\title{
Efficient Query Processing with Optimistically Compressed Hash Tables \& Strings in the USSR
}

\author{
Tim Gubner \\ CWI \\ tim.gubner@cwi.nl
}

\author{
Viktor Leis \\ Friedrich Schiller University Jena \\ viktor.leis@uni-jena.de
}

\author{
Peter Boncz \\ $C W I$ \\ peter.boncz@cwi.nl
}

\begin{abstract}
Modern query engines rely heavily on hash tables for query processing. Overall query performance and memory footprint is often determined by how hash tables and the tuples within them are represented. In this work, we propose three complementary techniques to improve this representation: Domain-Guided Prefix Suppression bit-packs keys and values tightly to reduce hash table record width. Optimistic Splitting decomposes values (and operations on them) into (operations on) frequently-accessed and infrequently-accessed value slices. By removing the infrequently-accessed value slices from the hash table record, it improves cache locality. The Unique Strings Selfaligned Region (USSR) accelerates handling frequently-occurring strings, which are very common in real-world data sets, by creating an on-the-fly dictionary of the most frequent strings. This allows executing many string operations with integer logic and reduces memory pressure.

We integrated these techniques into Vectorwise. On the TPC-H benchmark, our approach reduces peak memory consumption by $2-4 \times$ and improves performance by up to $1.5 \times$. On a real-world BI workload, we measured a $2 \times$ improvement in performance and in micro-benchmarks we observed speedups of up to $25 \times$.
\end{abstract}

\section{INTRODUCTION}

In modern query engines, many important operators like join and group-by are based on in-memory hash tables. Hash joins, for example, are usually implemented by materializing the whole inner (build) relation into a hash table. Hash tables are therefore often large and determine the peak memory consumption of a query. Since hash table sizes often exceed the capacity of the CPU cache, memory latency or bandwidth become the performance bottleneck in query processing. Due to the complex cache-hierarchy of modern CPUs, the access time to a random tuple varies by orders of magnitude depending on the size of the working set. This means that shrinking hash tables does not only reduce memory consumption but also has the potential of improving query performance through better CPU cache utilization [3], [4], [26].

To decrease a hash table's hunger for memory and, consequently, increase cache-efficiency, one can combine two orthogonal approaches: to increase the fill factor and to reduce the bucket/row size. Several hash table designs like Robin Hood Hashing [8], Cuckoo Hashing [23], and the Concise Hash Table [4] have been proposed for achieving high fill factors while still providing good lookup performance. Here, we investigate how the size of each row can be reduced-a topic that, despite its obvious importance for query processing, has not received as much attention.

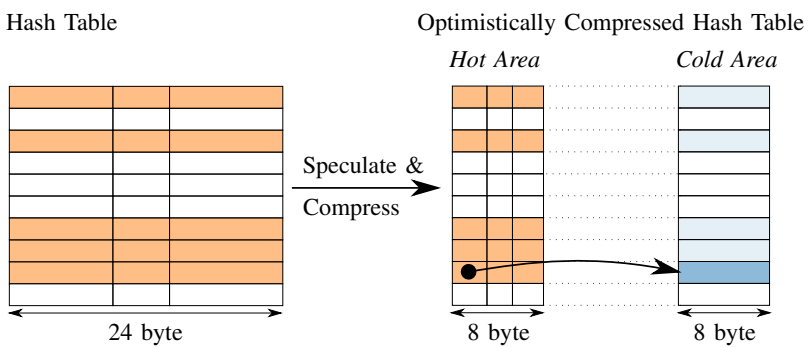

Fig. 1: Optimistically Compressed Hash Table, which is split into a thin hot area and a cold area for exceptions

While heavyweight compression schemes tend to result in larger space savings, they also have a high CPU overhead, which often cannot be amortized by improved cache locality. Therefore, we propose a lightweight compression technique called Domain-Guided Prefix Suppression. It saves space by using domain information to re-pack multiple columns inflight in the query pipeline into much fewer machine words. For each attribute, this requires only a handful of simple bit-wise operations, which are easily expressible in SIMD instructions, resulting in extremely low per-tuple cost (subcycle) for packing and subsequent unpacking operations.

Rather than saving space, the second technique, called Optimistic Splitting, aims at improving cache locality. As Figure 1 illustrates, it splits the hash table into a hot (frequentlyaccessed) and a cold (infrequently-accessed) area containing exceptions. These exceptions may, for example, be overflow bits in aggregations, or pointers to string data. By separating hot from cold information, Optimistic Splitting improves cache utilization even further.

An often ignored, yet costly part of query processing is string handling. String data is very common in many realworld applications [22], [31]. In comparison with integers, strings occupy more space, are much slower to process, and are less amenable to acceleration using SIMD. To speed up string processing, we therefore propose the Unique Strings Selfaligned Region (USSR), an efficient dynamic string dictionary for frequently-occurring strings. In contrast to conventional per-column dictionaries used in storage, the USSR is created anew for each query by inserting frequently-occurring strings at query runtime. The USSR, which has a fixed size of $768 \mathrm{kB}$ (ensuring its cache residency), speeds up common string operations like hashing and equality checks. 


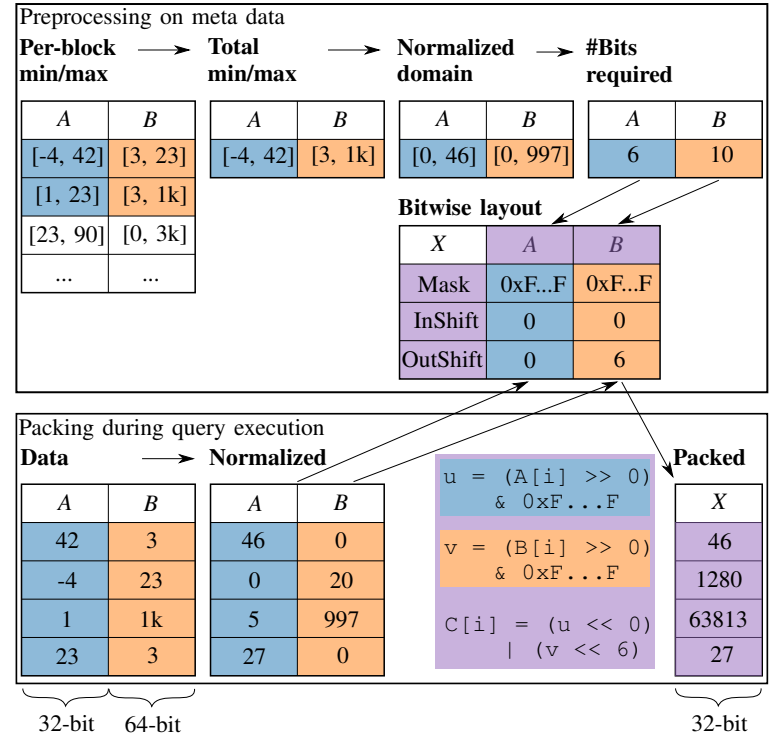

Fig. 2: Domain-Guided Prefix Suppression

While each of the proposed techniques may appear simple in isolation, they are highly complementary. For example, using all three techniques, strings from a low-cardinality domain can be represented using only a small number of bits. Furthermore, our techniques remap operations on wide columns into operations on multiple thin columns using a few extra primitive functions (such as pack and unpack). As such, they can easily be integrated into existing systems and do not require extensive modifications of the query processing algorithms or even hash table implementations.

Rather than merely implementing our approach as a prototype, we fully integrated it into Vectorwise which originated from the MonetDB/X100 project [7]. Describing how to integrate the three techniques into industrial-strength database systems is a key contribution of the paper.

Based on Vectorwise, we performed an extensive experimental evaluation using TPC-H, micro-benchmarks, and realworld workloads. On TPC-H, we reduce peak memory consumption by $2-4 \times$ and improve performance by up to $1.5 \times$. On a string-heavy real-world BI workload, we measured a $2.2 \times$ improvement in performance, and in micro-benchmarks we even observed speedups of up to $25 \times$.

\section{Domain-Guided PRefix SupPression}

Domain-Guided Prefix Suppression reduces memory consumption by eliminating the unnecessary prefix bits of each attribute. This enables us to cheaply compress rows without affecting the implementation of the hash table itself, which makes it easy to integrate our technique into existing database systems. In particular, while our system (Vectorwise) uses a single-table hash join, Domain-Guided Prefix Suppression would also be applicable (and highly beneficial) for systems that use partitioning joins [3], [28]. Domain-Guided Prefix Suppression also allows comparisons of compressed values without requiring decompression. In the rest of this section, we describe Domain-Guided Prefix Suppression in detail using Figure 2 as an illustration.

\section{A. Domain Derivation}

A column in-flight in a query plan can originate directly from a table scan or from a computation. If a value originates from a table scan, we determine its domain based on the scanned blocks. For each block we utilize per-column minimum and maximum information (called ZoneMaps or Min/Max indices). This information is typically not stored inside the block itself as this would require scanning the block (potentially fetching it from disk) before this information can be extracted. Instead, the meta-data is stored "outof-band" (e.g. in a row-group header, file footer or inside the catalog). By knowing the range of blocks that will be scanned, the domain can be calculated by computing the total minimum/maximum over the range.

On the other hand, if a value stems from a computation, the domain minimum and maximum can be derived bottom up according to the functions used, based on the minimum/maximum bounds on its inputs under assumption of the worst case. Consider, for example, the addition of two integers $a \in\left[a_{\min }, a_{\max }\right]$ and $b \in\left[b_{\min }, b_{\max }\right]$ resulting in $r \in\left[r_{\min }, r_{\max }\right]$. To calculate $r_{\min }$ and $r_{\max }$ we have to assume the worst-case that means the smallest $\left(r_{\text {min }}\right)$, respective highest $\left(r_{\max }\right)$, result of the addition. In case of an addition this boils down to $r_{\min }=a_{\min }+b_{\min }$ and $r_{\max }=a_{\max }+b_{\max }$.

Depending on these domain bounds, an addition of two 32bit integer expressions could still fit in a 32-bit result, or less likely, would have to be extended to 64-bit. This analysis of minimum/maximum bounds often can allow implementations to ignore overflow handling, as the choice of data types prevents overflow, rather than having to check for it. For aggregation functions such as SuM, overflow avoidance is more challenging but in Section III-A, we discuss Optimistic Splitting, which allows to do most calculations on small data types, also reducing the cache footprint of aggregates.

\section{B. Prefix Suppression}

Using the derived domain bounds, we can represent values compactly without losing any information by dropping the common prefix bits. To further reduce the number of bits and enable the compression of negative values, we first subtract the domain minimum from each value. Consequently, each bit-packed value is a positive offset to the domain minimum. We also pack multiple columns together such that the packed result fits a machine word. This is done by concatenating all compressed bit-strings, and (if necessary) chunk the result into multiple machine words. Each chunk of the result constitutes a compressed column which can be stored just like a regular uncompressed column.

Figure 2 shows an example where column $A$ contains values ranging from $d_{\min }=-4$ to $d_{\max }=42$. First, we transform values from that domain into non-negative integers by subtracting the domain minimum $(-4)$. These values can 
then be represented using $\left\lceil\log _{2}\left(d_{\max }-d_{\min }+1\right)\right\rceil=6$ bits. Afterwards we "glue" values from multiple columns together (here: $A$ and $B$ ) together.

\section{Compression and Decompression}

Like many modern column-oriented systems [1], Vectorwise is based on vectorized primitives that process cache-resident vectors (=arrays of single column values). These primitives process items from multiple inputs in a data-parallel (SIMDfriendly) fashion in a tight loop. Consequently, modern compilers automatically translate such code into SIMD instructions for the specified target architecture (e.g. AVX-512). In our vectorized hash table implementation, pack primitives compress and "glue" multiple inputs together to produce one intermediate result. Later, this intermediate result is then stored inside the hash table. With all the inputs and the one output being cache-resident vectors, the compression itself happens in-cache. For bit-packing, our pack primitives look similar to the following pseudo-code:

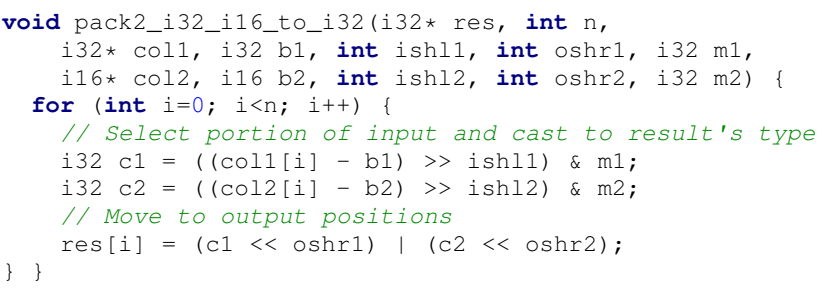

After bit-packing, we scatter the intermediate results into its final positions in the hash table. For improved cachelocality the hash table is stored in row-wise layout (NSM) [34]. Therefore we need to convert columnar vectors (DSM) into tuples (NSM). An interesting observation is that in NSM two subsequent column values are precisely one row width apart. Hence, for each attribute we calculate an attribute multiplier stride := row_width / attr_width which projects the row width onto the current attribute's width. When scattering attributes from vectors into ith NSM record, we calculate its final position $i *$ stride and copy each attribute to its respective position.

When decompressing values, we fetch up to 4 columns from the hash table and directly decompress them. For decompressing a vector of $\mathrm{n}$ packed 16-bit integers from 32-bit and 16-bit integers at positions $i d x$ in the hash table, this leads to the following pseudo-code (2-column example):

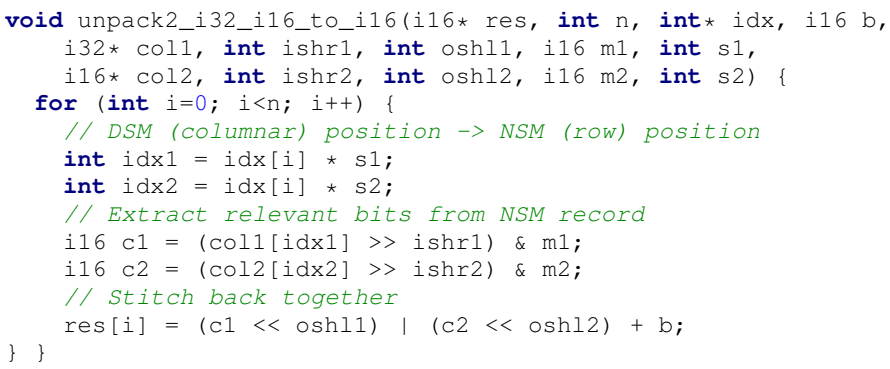

Notably compression and decompression operate in a nonintuitive fashion: Both process $m$ inputs and produce one output. This particular approach has two advantages: (a) In contrast to approaches with multiple outputs, it allows decompressing specific columns without enforcing decompression of neighboring cells. This allows an efficient mix of key checks on compressed data together with key checks on bit-packed non-integer data, most notably strings. (b) We concatenate bit-strings directly in registers, as opposed to approaches that partially compress/decompress which require multiple rounds of reading/writing from/to output vectors to concatenate partial output vectors into the final output.

We implemented our primitives similarly to the ones shown above and improved them further with "micro-adaptive" optimizations [27]. (1) Even though the implementation of pack supports selective processing, it can dynamically decide to process its inputs fully instead whenever $\geq 25 \%$ of the tuples in a batch are still active (selected); which favors SIMD. (2) In case all base values are zero, we avoid the integer subtraction, in case of pack, or addition, in case of unpack. This further reduces the number of required operations, if normalization is not required.

\section{Operating on Compressed Keys}

Domain-Guided Prefix Suppression also allows comparing compressed values themselves (without having to decompress). Assume key value $A$ is stored in the hash table and probe key $B$ is compared to $A$. Normally one would just fetch the key $A$ from the table and then compare it to $B$. In combination with compression, fetching $A$ also requires decompressing $A$. We argue it is better to first bring $B$ into the same representation as $A$, i.e., compressing $B$, and then directly compare the compressed values. This is especially true if keys $A$ and $B$ consist of multiple columns. For instance, a group-by on two columns can often be mapped into singleinteger compressed key, reducing computational work of hash aggregation (e.g. perform a single comparison, using fewer branches).

\section{E. Generating Pre-Compiled Kernels}

In order to integrate the on-the-fly compression, decompression, and checking routines into Vectorwise, we needed to generate pre-compiled kernels for each type combination. This means that we would have to generate kernels for up to $n+1$ types ( $n$ inputs, one output). For $n=8$ inputs, one output and 10 distinct types, we count $10^{8+1}$ kernels, leading to a heavily inflated binary file and - potentially—high lookup costs when resolving single kernels.

To reduce the number of generated kernels we (a) restrict the number of inputs to $\leq 4$. In addition we (b) restrict the types we pack into to 32-, 64- and 128-bit unsigned integers and (c) impose an order on the inputs (ordered by bit-width). All three restrictions limit the number of kernels that need to be generated to 3,000 pack kernels and 340 kernels that do decompression such as unpack-fetch or key checks. These kernels are generated using templating.

\section{F. Tackling the Packing Problem}

Prefix Suppression combines multiple prefix-suppressed codes from different attributes. However, in the vectorized 
execution model, we rely on pre-compiled primitives that only allow a fixed number of inputs (here $n=4$ ), to avoid a combinatorial explosion in the amount of functions needed. We must also choose the output data types of which the hash table record is made up (32 or 64-bit integers and rarely 128bit integers) and appropriately spread the compressed input columns in non-overlapping fashion over these base data types. In effect, we have to generate a packing plan, consisting of pack functions that (a) respects the maximum $n$ on pack input columns, (b) minimizes the total hash table record width in row-wise (NSM) layout as well as (c) minimizes the number of slices an input column is cut into.

For joins we separate the packing problem into two subproblems, one for packing the hash table key-columns only, and the second for packing all other columns. For aggregates, we only pack the key-columns. The other columns are aggregate results and they are left in their uncompressed layout. The reason is that packing and unpacking needed for every update to an aggregate result would slow down aggregations dramatically. In the next section, however, we describe a technique, called Optimistic Splitting, to shrink aggregates into smaller data types and, therefore, reduce the active working set, as well as CPU effort.

We pack the key-columns together, such that e.g. the TPC-H join on PARTSUPP will pack PS_PARTKEY and PS_SUPPKEY into one word, so we can execute the join as if there were just one column: this halves both hashing and comparison work. The algorithm is invoked twice: once packing into 32-bit words and once packing into 64-bit words. We use the 64-bit solution if this yields less hash table columns than the 32-bit solution, or otherwise, if the 64-bit solution produces a NSM record of the same size.

Greedy Packing Algorithm: The algorithm packs a set of columns, and first orders them in a queue $\mathrm{Q}$ on their bit-width (the bits needed after Domain-Guided Prefix Suppression). The sum of these bit-widths generally is less than a full multiple of the output word bit-size; let the amount of unused bits be $U$. The main round of the algorithm iteratively pops the largest column off $Q$ and checks if it fits the current output word. If not, it puts the column in the initially empty queue $Q^{\prime}$. Otherwise, it maps this column onto the current output word, hence reducing the amount of still unused bits $\mathrm{L}$ in this output word ( $L$ is initialized to the output word bit-width at the start of each round). When no column fits anymore and $Q=\emptyset$, we reset $Q=Q^{\prime}$ and $Q^{\prime}=\emptyset$ to move to a next round (output word). If at the end $L \leq U$ (there is free bit budget), then we simply decrement $U$ by $L$ and leave these bits free. Otherwise, the first popped column in the next round will be sliced: putting its highest unprocessed $L$ bits into the previous output word and starting the round with the rest of the column. The algorithm continues its rounds until all columns (or slices thereof) are mapped to bit ranges in the output words.

\section{Optimistic SPLITTING}

The goal of Optimistic Splitting is to exploit skewed access frequencies by separating the common case from exceptional
TABLE I: Optimistic Aggregates

\begin{tabular}{l|l|l} 
Aggregate & Common case & Exception \\
\hline SUM & Small integer & Overflow counter \\
MIN & Small upper bound & Minimum \\
MAX & Small lower bound & Maximum \\
\hline COUNT & \multicolumn{2}{|c}{ Similar to SUM } \\
AVG & Rewritten into $\frac{\text { SUM }}{\text { COUNT }}$
\end{tabular}

situations. We physically split the hash table into two areas: The frequently-accessed hot area and the cold area, which is accessed rarely. This approach does not necessarily save space. However it shrinks the active working set, leading to lower memory access cost. Also, it converts operations on the final, widest, data type into operations on a potentially smaller data type. Specifically, if 128-bit operations become 64-bit or 32-bit; this can speed up computation noticeably. As we show in the following, Optimistic Splitting is especially important for data that is hard to compress such as aggregates and strings.

\section{A. Optimistic Aggregates}

Aggregates are hard to compress with Domain-Guided Prefix Suppression as it is not possible to obtain tight bounds for aggregation results (for example sums). The reason is that one has to be pessimistic when deriving domain bounds to prevent integer overflows: Assuming a SUM of at most $2^{48}$ integers from say a 18-bit domain, would overflow 64-bit and thus need a 128-bit aggregate. If this type is used for the aggregate, on each addition in the sum this large 128-bit integer will be read, updated, and written back.

Using a 64-bit integer for the aggregate, on the other hand, would (a) reduce reads and writes by a factor 2 and (b) provide faster updates. Without sacrificing correctness, Optimistic Splitting allows one to do just that in the common case (i.e., when no overflow occurs): The 128-bit aggregate result is split into a frequently-accessed 64-bit sum and another, rarelyaccessed 64-bit overflow/carry field, which is stored separately. In pseudo code, this looks as follows:

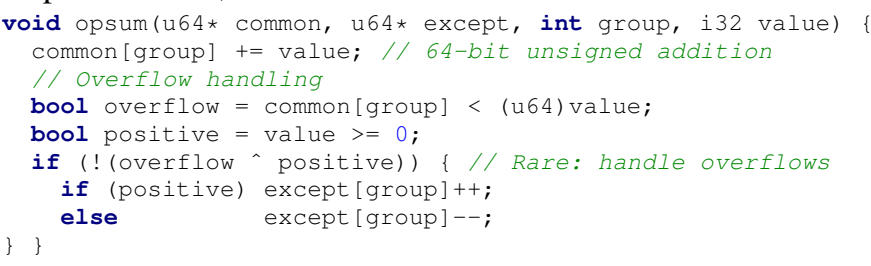

Note that this is a generic implementation that handles positive as well as negative values. In combination with domain bounds (Min/Max information) it is possible to prove the absence of negative or positive values which leads to simplified logic and improved performance. Our later micro-benchmarks show that this is an optimization that in many cases outperforms the full 128-bit sum.

Similarly, it is possible to shrink the working set of other aggregates. Table I illustrates how to exploit Optimistic Splitting for these. We use the associativity of aggregates to provide a fast path for large aggregates and a smaller working set. MIN can be implemented using an upper bound $(s)$ inside the hash table and storing the full minimum $e$ as an exception $(s \geq e)$. When calculating the aggregate one would first check against 
$s$ and discard values that cannot become the new minimum. For the remaining values one has to check against the full minimum and potentially update the full minimum $e$ as well as the upper bound $s$. Similar is the implementation of MAX whereas the other aggregate functions, COUNT and AVG, can be implemented similar to SUM. However, in case of COUNT one can more aggressively reduce the common case to a 16-bit integer and after $2^{16}-1$ iterations update both, the small optimistic counter as well as the exception.

\section{B. Other Applications}

Optimistic Splitting is a very general idea that we believe can be applied in many different use cases. It only requires that the entries of a hash table have different access patterns, and can be decomposed in some form.

Besides the aggregate decomposition approach described above, a second use case in the hash aggregation are functionally dependent group by keys. These are not touched by lookups, and can therefore be placed in the cold area.

Optimistic Splitting is also applicable to certain hash joins. For selective joins (i.e., where most probes are misses), only the keys need to be stored in the hot area, whereas the payload columns, which often occupy much more space than the keys, can be moved to the cold area.

Even the fact that the next pointer in chaining-based hash tables is often NULL can be exploited. A few bits in the hot area can indicate whether a next bucket is absent or is nearby. The full next pointer can reside in the cold area.

Finally, a weakness of the usage of global MinMax information, is that outliers can destroy the tight MinMax bounds that would capture most of the value distribution. Alternatively, one could derive MinMax bounds from a table sample. The hot area in hash tables would hold the "Sample-Guided" Prefix Suppressed values extended with an exception bit. The full uncompressed columns would be present in the exception area, but only be accessed for the outlier values.

These examples show that Optimistic Splitting is widely applicable. We also think that some implementation techniques could be further developed. For instance, for aggregates with few groups (and certainly global aggregates, without grouping), vectorized systems could keep more aggressive overflow bounds that guarantee that a batch of aggregate updates cannot overflow the partial aggregate. This way, overflow checking could be done once per vector, rather than for every tuple.

We defer investigation of these ideas to future work.

\section{USSR: A DYNAMIC STRING DICTIONARY}

Strings are prevalent in many real-world data sets [14], [22], [31] and present additional challenges for query performance. In contrast to integers, any individual string generally does not fit into a single CPU register and requires multiple instructions for each primitive operation (e.g. comparison). Strings are also often larger than integers, which negatively affects memory footprint and cache locality. Furthermore, neither Domain-Guided Prefix Suppression nor Optimistic Splitting can directly be applied to strings. This section presents a

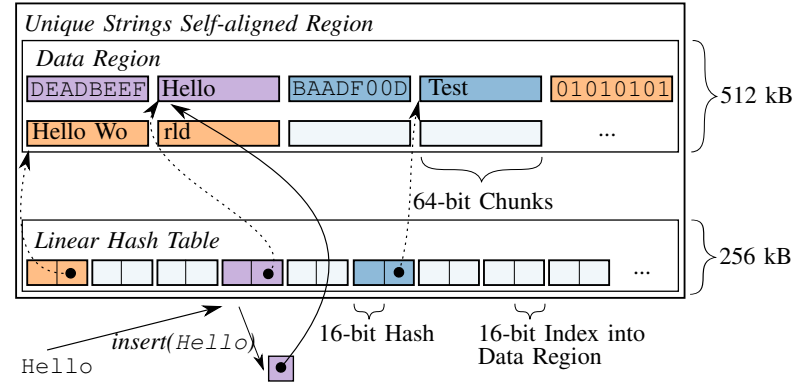

Fig. 3: Unique Strings Self-aligned Region (USSR) data structure details

dynamic data-structure called Unique Strings Self-aligned Region, which saves memory and enables processing strings at almost the same speed as integers.

\section{A. The Problems with Global Dictionaries}

To improve the performance of strings, some main-memory database systems - most notably SAP HANA [10]—represent strings using per-column dictionaries where codes respect the value order. Using these dictionaries, string comparisons and hashing operations can be directly performed on the dictionary keys, which are fixed-size integers, rather than variable-length strings. Unfortunately, global dictionaries have significant downsides, which have precluded their general adoption. First, because random access to the dictionaries is common, the dictionaries must fully reside in main memory. For systems that must manage data sets larger than main memory (e.g. analytical column stores), this is a major problem. Also, systems that support parallel and distributed execution, including those designed or optimized for the cloud, face the problem that bulk-loading or updating tables in parallel would require continuous synchronization in order to maintain a consistent global dictionary. Another downside is that dictionaries incur significant overhead for inserts, updates, and deletes-in effect they are a mandatory secondary index on every string column. If, for instance, new values appear, extending the dictionary such that one additional bit is needed to represent a code, updates will no longer fit in previously encoded data. Deletes of no longer used strings leave holes in the code space that need to be garbage collected and inserts in sorted dictionaries often require re-coding-which involves fully rewriting all encoded columns periodically.

Given these problems with global dictionaries, most database systems therefore limit themselves to per-block dictionaries (e.g. one dictionary for every 10,000 strings). With this approach, dictionaries are a local feature mainly used for compression rather than a global data structure. Per-block dictionaries are often almost as space-effective as per-column dictionaries without sharing their in-memory limitations and update overheads. For query processing, however, the advantage of per-block dictionaries is limited. While some systems evaluate pushed-down selections directly on the dictionary [15], all other operations require decompression and therefore do not benefit from the dictionary. The reason is that the dictionary 
is only available to the table scan operators. Materializing operators like hash join and aggregation therefore typically allocate memory on the heap for every string. Needless to say, this is very inefficient, yet for some reason dealing with strings is only a sparsely researched topic.

\section{B. Unique Strings Self-aligned Region (USSR)}

The Unique Strings Self-aligned Region is a query-wide data structure that contains the common strings of a particular query. In contrast to the heap, all strings within the USSR are known to be unique, which enables fast operations on these strings. To make it cache resident and efficient, the USSR has a limited size. Once it is full, strings need to be allocated on the heap as usual. By removing duplicates in this opportunistic fashion, the USSR reduces the number of heap allocations and therefore minimizes peak memory consumption.

By default, both heap-backed and USSR-backed strings are represented as normal pointers, which means that query engine operators can treat all strings uniformly without any code modifications. This allows to retro-fit this idea easily into already existing engines. However, by exploiting the dictionary-like nature and artful implementation of the USSR, the following additional optimizations become possible for USSR-based strings: (a) String comparisons are almost as fast as integer comparisons. (b) Hashes are pre-calculated and stored within the USSR, speeding up hash-based operators like join and group by. (c) Since the size of the USSR is limited, frequent strings can also be represented using small integer offsets, which can be exploited e.g. in Optimistic Splitting.

To summarize, the USSR is a lightweight, dynamic, and opportunistic string dictionary. It does not require changes to the storage level but is implemented in the query processor, and speeds up queries with low to medium string cardinalities, which is where global string dictionaries excel.

\section{Data Structure Details}

Our USSR implementation limits its capacity to $768 \mathrm{kB}$ : it consists of a hash table $(256 \mathrm{kB})$ and a data region $(512 \mathrm{kB})$. Figure 3 serves as an illustration of the USSR.

The $512 \mathrm{kB}$ data region starts at a self-aligned memory address (i.e., the pointer has $0 \mathrm{~s}$ in its lowest 19 bits). If one allocates $1 \mathrm{MB}$ of data, there is always a self-aligned address in its first half for the data region; and there is always either $256 \mathrm{kB}$ space before or after the data region for the hash table. The self-aligned memory address guarantees that all pointers inside the data region start with the same 45 bits prefix. This allows to very efficiently test whether a string pointer points inside the USSR (by applying a mask).

The data region stores the string data and materializes the string's hash value just before it. These numbers are stored aligned, so the data region effectively consists of $64 \mathrm{k}$ slots of 8 bytes where a string can start. Given that each string takes at least two slots (one for the hash and one for the string) the USSR can contain maximally $32 \mathrm{k}$ strings.

When inserting a string, the USSR needs to check whether that string is already stored, and if so, return its address rather than insert a new string. To do this in low $O(1)$, there is a fast linear probing hash table, consisting of 64k 4-byte buckets. Each bucket consists of a 16-bit hash extract and a 16-bit slot number that points into the data region to the start of the string. The lowest 16-bits of the string hash are used for locating the bucket, and the next 16-bits are the extract used to quickly identify collisions. The load factor is always below 50\% (64k buckets for at most $32 \mathrm{k}$ strings).

\section{Insertion}

The purpose of the USSR is to accelerate operations on frequent strings. In the extreme, all strings could be part of the USSR. However, due to its limited size, the USSR can only fit a sample. The sampling happens during insertion into the data structure. Failure during insertion might happen because (a) the string is rejected based on our sampling strategy or (b) a probing sequence of longer than 3 in the linear hash table is detected (due to the low load factor, this is highly infrequent, yet keeps negative lookups fast).

Our sampling strategy gives priority to string constants that occur in the query text; these are inserted first. After that, scans will insert strings until the USSR is full. We argue that the fact that a string column is dictionary-compressed, indicates that strings stem from a domain with a small cardinality. Therefore these strings are good candidates for insertion into the USSR.

Vectorwise stores and buffers data in compressed form and decompresses column slices on the fly in the table scan operator. When reading a new dictionary-compressed block, the scan needs to set up an in-memory array with string pointers. Strings are represented as pointers in-flight in a query and decompression means looking up dictionary codes into this array. Rather than pointing into the dictionary inside the buffered block, when setting up this array, the scan inserts all dictionary strings into the USSR, so (most of) these pointers will point into the USSR instead. Insertion may fail, in which case the pointers still point into the block.

The sampling strategy further tries to optimize usage of the limited data region, by failing inserts of long strings that occupy $>\min \left(F, \max \left(2,\left\lfloor\frac{F}{64}\right\rfloor\right)\right)$ 8-byte slots, where $F$ is the free space in the data region (in slots). The idea is that it is better to accept more small strings than a few large strings, in case space fills up.

\section{E. Accelerating Hashing \& Comparisons}

The USSR can be used to speed up hash computations. After testing whether a given string resides in the USSR using a bitwise and operation, one can directly access the pre-computed hash value, which physically precedes the string:

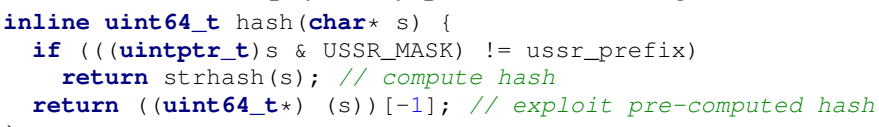
\}

The USSR also speeds up string comparisons when both compared strings reside in it. We exploit the fact that all strings within the USSR are unique. Hence, if the pointers are equal, the strings themselves are: 


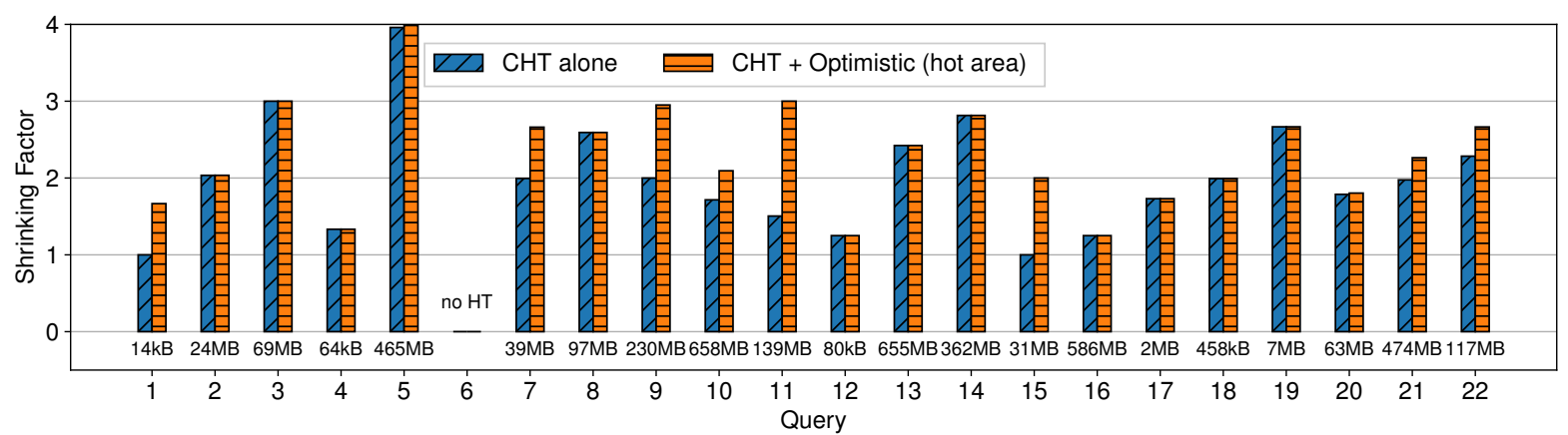

Fig. 4: Reduction in hash table memory footprint over TPC-H power run with absolute baseline hash table memory footprint (below the bar)

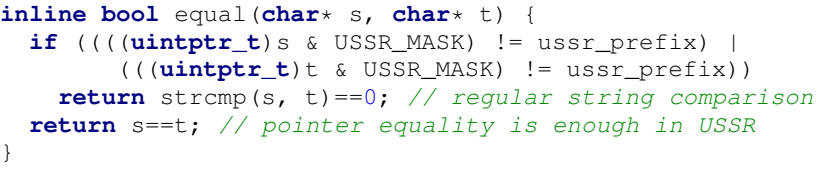

\section{F. Optimistic Splitting \& the USSR}

Optimistic Splitting and the USSR are complementary. The idea is to store USSR-backed strings, as small integers, compactly in the hot area and heap-backed strings in the cold area. Specifically, rather than storing string pointers in the hot area, we store slot numbers, pointing into the USSR. As mentioned earlier, these slot numbers are limited to $2^{16}$, so they can be represented as unsigned 16-bit integers .

During packing, we represent exceptions using the invalid slot number 0 in the hot area of the hash table, and store the full 64-bit pointer in the exception area. Whenever a string needs to be unpacked, we first access the hot area and unpack the slot number. For non-zero slot numbers we can directly reconstruct the pointer of the string (base address of USSR data region $+s l o t * 8)$. However, we can further accelerate equality comparisons on strings by first comparing the slot numbers and, only if they are 0 , comparing the full strings. A USSR encoded string $\mathrm{p}$ can be translated into a slot number quickly using ( $p>>3) \& 65535$.

\section{EXPERIMENTAL EVALUATION}

In this section, we provide an experimental evaluation of our contributions to show that our techniques improve performance as well as memory footprint.

For this evaluation, we integrated Domain-Guided Prefix Suppression, Optimistic Splitting, and the USSR into Vectorwise. Besides generating all necessary function kernels, we had to extend the domain derivation mechanism and implement our greedy packing algorithm. In addition, we modified the existing hash table implementation, extended the hash join operator to take advantage of compressed key and payload columns, as well as the hash aggregation (group by) operator to support Optimistic Aggregates.

We first evaluate the end-to-end performance on the TPC$\mathrm{H}$ benchmark. We then present a high-level comparison on a real BI workload from Tableau Public [31]. Next, we selected queries from the workload, provide a detailed breakdown and explain the impact of our techniques. Afterwards we move to micro-benchmarks, analyze and discuss the impact of the USSR on string-intensive queries. Then we evaluate the hash probe performance over varying hash table sizes and the influence of different domains on hash table performance. Afterwards we present and discuss the performance of our compression kernels, followed by an evaluation of Optimistic sum aggregates.

All experiments were performed on a dual-socket Intel Xeon Gold 6126 with 12 physical cores and 19.25 MB L3 cache each. The system is equipped with 384 GB of main memory. All results stem from hot runs using single-threaded execution.

\section{A. TPC-H Benchmark}

We evaluated the impact of Domain-Guided Prefix Suppression, Optimistic Splitting and the USSR on the widelyused TPC-H benchmark with scale factor 100 . We executed all 22 queries on our modified Vectorwise with and without our optimizations. We measured hash table memory footprint, as well as query response time. First, we present and discuss the performance regarding memory footprint and, afterwards, query performance.

Memory Footprint: In Vectorwise the memory consumption of many queries, particularly the TPC-H queries, is dominated by the size of hash tables. Therefore, during the TPC-H power run, we measured hash tables sizes. Figure 4 shows the compression ratios we measured.

Domain-Guided Prefix Suppression (CHT alone), without Optimistic Splitting and USSR, was able to reduce hash table size by up to $4 \times$. However, due to certain hurdles the compression ratio is often limited to $2 \times$ :

(a) Aggregates are not compressible without Optimistic Splitting. (b) Without the USSR, each string has to be a 64-bit pointer into a string heap. On recent hardware this requires storing at least 48 bits with Domain-Guided Prefix Suppression. (c) As CHT does not make sense for CPU cacheresident hash tables, we do not enable it if the hash table is small, based on optimizer estimates.

The impact of (a) and (b) on the active working set will be reduced using Optimistic Splitting and the USSR.

Optimistic Splitting aims at improving performance through more efficient cache utilization by separating the hash table 
TABLE II: Reduction in hash table memory footprint on TPC-H comparing vanilla Vectorwise against optimistically compressed hash tables including hot and cold area

\begin{tabular}{ccccccccccccccccccccccc}
\hline Query & 1 & 2 & 3 & 4 & 5 & 6 & 7 & 8 & 9 & 10 & 11 & 12 & 13 & 14 & 15 & 16 & 17 & 18 & 19 & 20 & 21 & 22 \\
\hline Factor & 0.8 & 1.6 & 1.5 & 1.0 & 2.0 & 0.0 & 1.1 & 2.0 & 1.4 & 1.4 & 1.0 & 1.0 & 1.4 & 2.1 & 1.0 & 1.0 & 1.3 & 1.6 & 1.6 & 1.3 & 1.4 & 1.6 \\
\hline
\end{tabular}

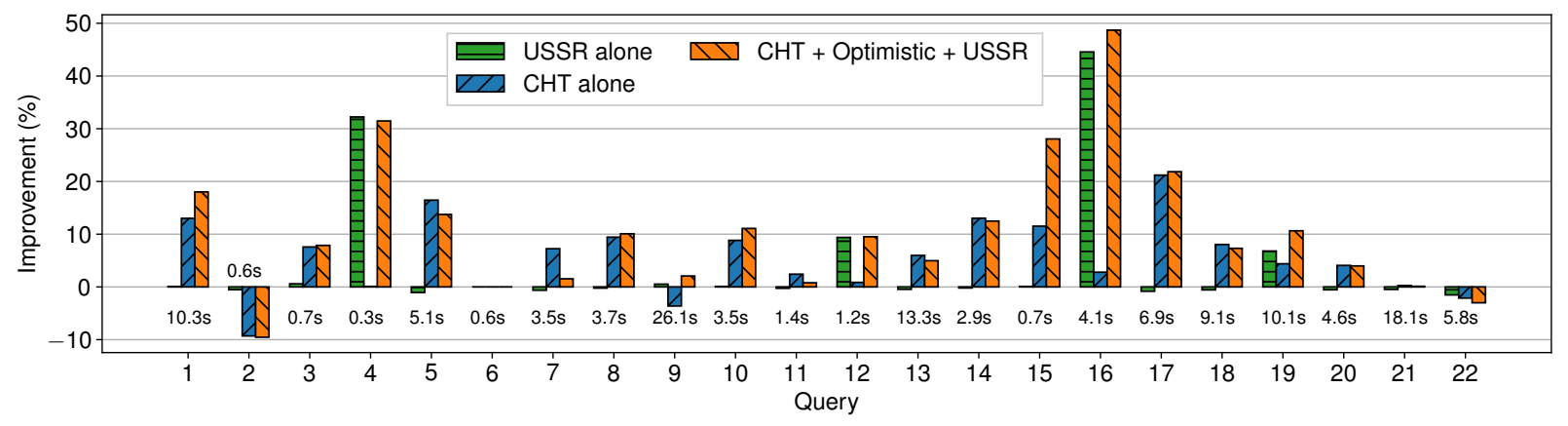

Fig. 5: Improvement over TPC-H power run with baseline times (under the bar)

into a thin frequently-accessed table (hot area) and a rarely accessed table (cold area). In combination with the USSR we measured a $2-4 \times$ smaller hot area $(C H T+$ Optimistic (hot area)) in many TPC-H queries.

However, Optimistic Splitting in fact increases (rather than reduces) the overall memory consumption as it introduces additional data. For example, splitting a 128-bit sum aggregate will introduce an additional aggregate with a smaller size but the full 128-bit aggregate will still reside in cold area. Table II shows the relative memory footprint of vanilla Vectorwise against the combination of Domain-Guided Prefix Suppression, Optimistic Splitting and the USSR. Over TPC-H we measured up to $2.1 \times$ lower memory consumption. However, in comparison to Domain-Guided Prefix Suppression alone, Optimistic Splitting achieves an inferior compression ratio. The main idea behind Optimistic Splitting is to reduce memory pressure rather than overall memory consumption.

Query Performance: To demonstrate the performance benefits of the USSR, Domain-Guided Prefix Suppression and Optimistic Splitting, we visualize the query response times of all 22 TPC-H queries in Figure 5 We split our analysis into three stages. First, we evaluate the impact achieved by only using USSR. Then we discuss the effects of only using Domain-Guided Prefix Suppression. Finally, the influence of the combination of all three techniques will be discussed.

The idea of the USSR is to boost operations on frequent strings. However, TPC-H is not an extremely string-intensive benchmark. Nonetheless, by using the Unique Strings Selfaligned Region (USSR alone) three queries (Q4, Q12 and Q16) showed significant performance gains. All three benefit from faster string hashing and equality comparisons provided by the USSR and improve by up to $45 \%$.

Apart from the string-specific USSR, Domain-Guided Prefix Suppression aims at shrinking hash tables and providing operations on compressed data. We found that Domain-Guided Prefix Suppression accelerates most queries (CHT alone) by up to $30 \%$. In most queries we noticed an improvement of at least $10 \%$. This is caused by the more efficient expression evaluation that smaller data types provide and the more cacheefficient hash table that allows equality comparisons directly on compressed keys. Notably, the regression in Q2 was caused by type casting overhead which occurred when operating on compact data types. We highlight that the purpose of DomainGuided Prefix Suppression is mostly to reduce the memory footprint and not necessarily to speedup query evaluation.

When combining all three techniques (Domain-Guided Prefix Suppression, USSR and Optimistic Splitting) we measured gains up to $40 \%(C H T+$ Optimistic + USSR $)$. We measured additional improvements from $5 \%$, in Q1, up to $10 \%$, in Q15. Both queries benefited from the Optimistic SUM aggregate which boosted the aggregate computation.

We ran all 22 queries with intra-query parallelism and noticed similar performance improvements. However, as these runs were considerably more noisy and would not contribute significant new information, we excluded them from this paper.

\section{B. Public BI Benchmark}

It has been noted that synthetic benchmarks like TPC-H do not capture all relevant aspects of real workloads [6], [9]. Recently, a workload study was published [31] based on the Tableau Public ${ }^{1}$ Business Intelligence (BI) free cloud service. It analyzes its workbooks (data and queries generated by the Tableau BI tool) and specifically notes that users make extensive use of string data types (i.e., strings are by far the most common data type; used for $49 \%$ of all values). Not only is text data prevalent in these workbooks, but it is also observed that date columns, numeric and decimal columns are often stored as strings; arguably sub-optimally, but often this is related to data cleaning issues. Regrettably, this study did not publish the data and queries as an open benchmark, also upon our request to Tableau. Inspired by this work, we manually downloaded the 48 biggest Tableau Public workbooks (400 GB data) and extracted the SQL statements from its query log. This workload is now available in open-source as the Public

\footnotetext{
${ }^{1}$ https://public.tableau.com
} 
TABLE III: Speedup and USSR statistics for workbook CommonGovernment

\begin{tabular}{|c|c|c|c|c|c|c|c|c|c|c|c|c|c|c|c|c|c|c|c|c|}
\hline Query & 1 & 2 & 3 & 4 & 5 & 6 & 7 & 8 & 9 & 10 & 11 & 12 & 13 & 14 & 15 & 16 & 17 & 18 & 19 & 20 \\
\hline Speedup & 2.1 & 1.4 & 2.2 & 1.4 & 1.3 & 1.0 & 1.2 & 1.0 & 1.5 & 1.8 & 1.1 & 2.2 & 2.2 & 1.8 & 1.5 & 2.1 & 1.4 & 1.1 & 1.4 & 1.1 \\
\hline USSR Size $(\mathrm{k}$ & 1.8 & 0.5 & 2.0 & 0.3 & 66.1 & 512.0 & 83.2 & 512.0 & 12.7 & 7.2 & 112.4 & 1.9 & 1.8 & 7.2 & 1.8 & 2.0 & 1.8 & 110.3 & 0.3 & 512.0 \\
\hline Rejection Ratio (\%) & 0.0 & 0.0 & 0.0 & 0.0 & 0.0 & 18.3 & 0.0 & 32.8 & 0.0 & 0.0 & 0.0 & 0.0 & 0.0 & 0.0 & 0.0 & 0.0 & 0.0 & 0.0 & 0.0 & 21.1 \\
\hline \#Rejected & $\overline{0}-$ & $0^{-}$ & $\overline{0}-$ & $\overline{0}^{-}$ & $\overline{0}$ & $\overline{3} 7 \overline{6} \overline{27}-$ & $0^{-}$ & ${ }^{-} 3 \overline{0} 2 \overline{0} 4^{-}$ & $\overline{0}$ & $-0^{-}$ & $0^{-}$ & $0^{-}$ & $\overline{0}$ & $0^{-}$ & $\overline{0}$ & $0^{-}$ & $\overline{0}$ & $-1 \overline{7}$ & $0^{-}$ & $\overline{1} 3 \overline{\overline{7}} 4 \overline{2}$ \\
\hline \#Candidates & 1312 & 2720 & 1056 & 1504 & 73200 & 205440 & 77296 & 92208 & 656 & 6768 & 110704 & 1072 & 1232 & 6752 & 3792 & 1360 & 3808 & 99744 & 1728 & 65222 \\
\hline \#Strings in USSR & 46 & 16 & 49 & 11 & 2251 & 12227 & 2835 & 12218 & 252 & 165 & 3041 & 48 & 45 & 167 & 51 & 49 & 51 & 2990 & 11 & 21343 \\
\hline Average String Lengt & 23 & 3 & 20 & 5 & 18 & 26 & 19 & 29 & 21 & 25 & 23 & . & 22 & 25 & 18 & 2 & 19 & 23 & 5 & 11 \\
\hline Baseline Rur & 0.15 & 0.27 & 0.13 & 0.17 & 0.37 & 3.48 & 0.39 & 0.54 & 0.18 & 0.16 & 0.29 & 0.14 & 0.14 & 0.16 & 0.18 & 0.14 & 0.1 & 0.27 & 0.18 & 0.51 \\
\hline Baseline HT size (MB) & 0.05 & 0.09 & 0.05 & 0.09 & 0.14 & 82.11 & 0.14 & 9.12 & 0.07 & 0.05 & 0.11 & 0.05 & 0.05 & 0.05 & 0.05 & 0.05 & 0.05 & 0.11 & 0.09 & 8.11 \\
\hline
\end{tabular}



Fig. 6: Query time breakdown for selected queries of workbook CommonGovernment

BI Benchmark As a representative example, we focus on one of its workbooks: CommonGovernment.

We extracted all 43 queries and all 13 tables. Each table contains around $8 \mathrm{GiB}$ of data in CSV format. Unlike TPC$\mathrm{H}$, each table contains many string columns and columns that contain NULL values are common. We executed each query sequentially and Table [III shows the measured effects on the runtime.

The workbook CommonGovernment is string-intensive: using only the USSR, we measured a speedup of up to $\approx 2 \times$ (55\% improvement). These speedups are caused by the fact that on the one hand many strings originate from a small domain of unique strings and thus become resident in the USSR. On the other hand, many strings are long enough to significantly impact string operations to cause a speedup of the whole query.

Q6, Q8 and Q20 show no significant benefits from the USSR mainly because the string columns have a large unified dictionary (that does not even fit fully in the USSR). While dictionary-coded decompression in Vectorwise has a sub-cycle per/tuple cost, the effort of setting up the dictionary array when the scan moves to a new disk block increases, when the per-block dictionary size increases. With the USSR, this setting-up effort becomes significantly higher as all dictionary strings must be looked up in the USSR linear hash table. Also, with larger dictionaries per block, each dictionary string has a lower repetition count during execution; so the amortization of the setting-up investment by faster hashing and comparison decreases. Still, we see that we make a good trade-off, as queries Q8 and Q20 still get (marginally) faster, and only Q6 is marginally slower.

\footnotetext{
${ }^{2}$ https://github.com/cwida/public bi benchmark
}

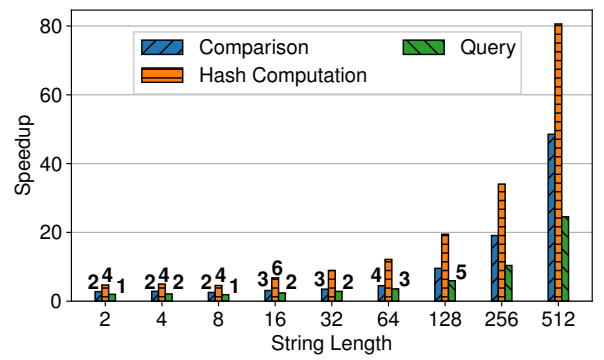

Fig. 7: Group-By on string keys: Speedup vs. length

In general, the Public BI workload is characterized by few joins and many aggregations [31], where these aggregations produce small results-few or in the thousands, but almost never in the millions of tuples. This means that the hash tables needed for aggregation are often CPU cache-resident. Therefore, CHT is not triggered and that the USSR is what most matters in this workload, so we focus only on that.

Breakdown: On the string-intensive workbook CommonGovernment the USSR caused many queries to improve up to $\approx 2 \times$. Figure 6 shows a breakdown of the query time for Q1, Q2 and Q4. For Q1 we now discuss how close $2 \times$ comes to the optimal speedup: The USSR led to a roughly $7 \times$ faster hash computation and a $2 \times$ faster key check (check whether keys are equal). This led to a total speedup of $2 \times$. Assuming zero cycles would be spent on hash computation and key checks, then one could achieve a total of $3 \times$. This, however, is a theoretical optimum and is unlikely to be achieved when performing these operations.

\section{Micro-Bench: USSR and Group-By}

We now move to a number of micro-benchmarks to focus on individual performance aspects of string processing with the USSR. We start with the performance on a SELECT COUNT (*) FROM T GROUP BY $S$ query. These strings came from a domain of 10 unique strings, all strings had the same length. Figure 7 shows the speedups that can be achieved using the USSR. We profiled the time spent on string comparisons when checking the keys inside group by's hash table. This results show significant speedups reaching from a $2 \times$ to $50 \times$ faster string comparison. Similarly, we profiled the time spent on computing hash of the string keys. This results in speedups reaching from $4 \times$ for small strings, to $80 \times$ for large strings.

Besides the significant speedup in terms of string comparison and hash computation, we also noticed significant speedup of the whole query up to $\approx 25 \times$. 


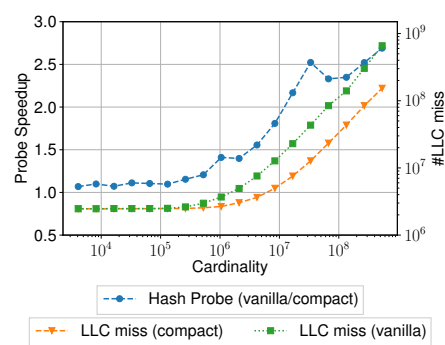

(a) 4 keys $k_{1}, \ldots, k_{4} \in[0,1.000]$ whereas schema suggests 64-bit integers

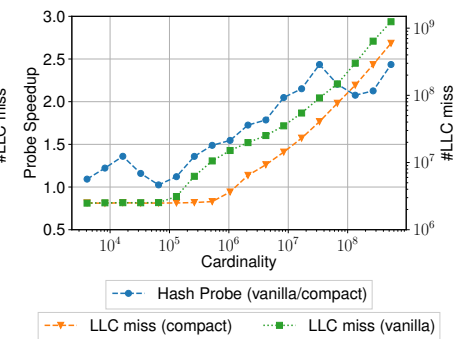

(b) 2 keys $k_{1}, k_{2} \in\left[0,10^{6}\right]$ whereas schema suggests 128bit integers

Fig. 8: Hash probe speedup vs. build-side cardinality using 4 payload columns $p_{1}, \ldots, p_{4} \in[0,10]$

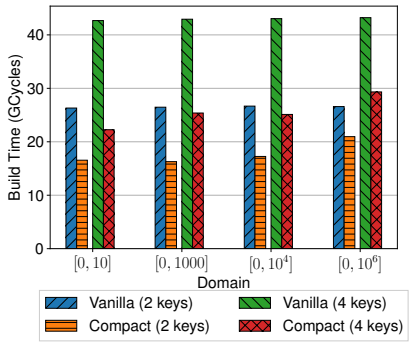

(a) Build time



(b) Hash table size
Fig. 9: Hash join performance vs. key domain

\section{Micro-Bench: Join Probe Performance}

We now micro-benchmark Domain-Guided Prefix Suppression, with respect to hash table lookup performance. Our experiment consists of a simple join query where we vary the size of the inner/build relation and the domains of the key columns. We experimented with two and four key columns, four payload columns with values $v \in[0,10]$.

Figure 8 visualizes the speedup, as well as, the L3/lastlevel cache (LLC) misses measured. We observe an up to $2.5 \times$ faster hash probe including the tuple reconstruction cost. The measured speedups tend to increase with hash table size (size of inner relation). For large hash tables with more than $10^{6}$ rows the speedups were caused by the significantly smaller and, consequently, more cache-resident hash table. For hash tables with less than $10^{6}$ rows performance was mostly affected by the more efficient comparisons directly on compressed data.

\section{E. Micro-Bench: Hash Join Key Domain}

The compression ratio and access performance of DomainGuided Prefix Suppression depends on the domain of the input values. We now micro-benchmark the effects of input domain(s) on hash join build time and compression ratio. We executed a simple (hash) join query with a large fixed-size inner (build) relation over multiple domains, with multiple keys ( 2 and 4 ) and without any payload columns. We measured build time, as well as, hash table size.

First, we investigated the impact of Domain-Guided Prefix Suppression on the build phase. Figure 9a shows the results.
TABLE IV: Reduction in memory footprint of our compressed hash table compared to other hash table designs. With $n$ values $\in\left[0,2^{16}\right)$ and $50 \%$ fill rate. Higher is better.

\begin{tabular}{|c|c|c|c|c|c|c|c|c|}
\hline & \multicolumn{7}{|c|}{ \#64-bit Values } \\
\hline & & 1 & 2 & 4 & 8 & 16 & 24 & 32 \\
\hline \multicolumn{9}{|c|}{ Linear Hash Table } \\
\hline \multirow{11}{*}{ 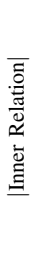 } & $1 \mathrm{k}$ & $2.0 \times$ & $3.2 \times$ & $4.6 \times$ & $5.8 \times$ & $6.7 \times$ & $7.1 \times$ & $7.3 \times$ \\
\hline & $1 \mathrm{M}$ & $1.1 \times$ & $2.0 \times$ & $3.2 \times$ & $4.6 \times$ & $5.8 \times$ & $6.4 \times$ & $6.7 \times$ \\
\hline & $1 \mathrm{G}$ & $1.1 \times$ & $2.0 \times$ & $3.2 \times$ & $4.6 \times$ & $5.8 \times$ & $6.4 \times$ & $6.7 \times$ \\
\hline & Con & se Has & Table 4 & & & & & \\
\hline & $1 \mathrm{k}$ & $1.1 \times$ & $1.6 \times$ & $2.3 \times$ & $2.9 \times$ & $3.4 \times$ & $3.6 \times$ & $3.7 \times$ \\
\hline & $1 \mathrm{M}$ & $0.6 \times$ & $1.0 \times$ & $1.6 \times$ & $2.3 \times$ & $2.9 \times$ & $3.2 \times$ & $3.4 \times$ \\
\hline & $1 \mathrm{G}$ & $0.6 \times$ & $1.0 \times$ & $1.6 \times$ & $2.3 \times$ & $2.9 \times$ & $3.2 \times$ & $3.4 \times$ \\
\hline & $\mathrm{Buc}$ & t-chain & Hash T & able & & & & \\
\hline & $1 \mathrm{k}$ & $1.8 \times$ & $2.2 \times$ & $2.7 \times$ & $3.2 \times$ & $3.5 \times$ & $3.7 \times$ & $3.7 \times$ \\
\hline & $1 \mathrm{M}$ & $1.4 \times$ & $1.8 \times$ & $2.2 \times$ & $2.7 \times$ & $3.2 \times$ & $3.4 \times$ & $3.5 \times$ \\
\hline & $1 \mathrm{G}$ & $1.4 \times$ & $1.8 \times$ & $2.2 \times$ & $2.7 \times$ & $3.2 \times$ & $3.4 \times$ & $3.5 \times$ \\
\hline
\end{tabular}

We noticed a pronounced increase in performance ranging from $25 \%$ up to $2 \times$.

As mentioned, the performance of Domain-Guided Prefix Suppression depends on the compression ratio. Figure $9 b$ shows the hash table size with and without our compression technique. For small domains, here $[0,10]$ and $[0,1000]$, we notice a compression ratio from $2 \times$ to $2.5 \times$. With two keys the compression ratio is limited by the output type size of our transformation where we have to round up to that type's size. Using more keys, we can fill the output word more densely and, hence, achieve a higher compression ratio up to $2.5 \times$. For larger domains, $\left[0,10^{6}\right]$, our scheme cannot achieve a high compression ratio, since there are simply not enough redundant 0-bits to suppress.

\section{F. Micro-Bench: Memory Footprint against other Hash Tables}

We benchmarked the memory footprint of our compressed hash tables against linear, Concise [4] and uncompressed bucket-chained hash tables. Table IV shows the reduction in memory footprint.

We observed that especially for large and wide hash tables, common in analytical queries, our compressed hash table easily out-performs the three other designs by $\approx 2 \times$ to $7 \times$. For extremely thin hash tables, our compressed bucket-chained hash table, shows additional overhead as next pointer, as well as bucket pointer needs to be stored. However, this is merely a limitation of our implementation. By applying Domain-Guided Prefix Suppression to the Concise Hash Table, one could easily construct a much more memory-efficient hash table.

\section{G. Micro-Bench: Compression Overhead}

Our next micro-benchmark showcases that Domain-Guided Prefix Suppression is very lightweight and compression overhead for common types is negligible. The compression performance is visualized in Figure 10. For native integer types, namely 8, 16, 32 and 64-bit, our implementation is able to compress between 1 and 2 output values per cycle. In contrast, packing 128-bit integers is significantly slower. However, 128bit integers rarely occur in real-world data sets and mainly stem from aggregates, for which we use Optimistic Splitting (such that packing/unpacking 128-bits values is not needed). 


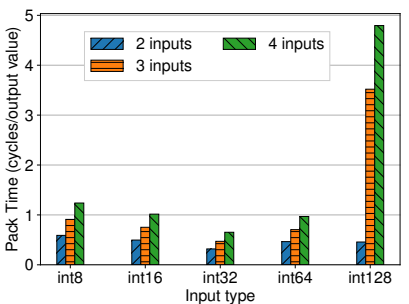

(a) Packing values into 32-bit integers



(b) Packing values into 64-bit integers
Fig. 10: Compression performance of bit-packing the first 8 bits of each input

\section{H. Micro-Bench: Optimistic Splitting}

Finally, we micro-benchmark the effectiveness of Optimistic Splitting for SUM aggregates. We evaluated the performance of 128-bit optimistic SUM in the following experiment:

We sum up 64-bit integers into a 128-bit aggregate. We implemented a vectorized "full" aggregation that adds each 64bit value into the 128-bit aggregate and an optimistic version with a 64-bit partial aggregate. Each vectorized aggregation primitive processes cache-resident vectors of input values, indices, aggregates and data. We used $2^{32}$ input values each equal to a constant $x$. Each value is then summed up per group. To control overflows, we varied the constant $x$ and the number of groups. All aggregates (full and partial) are stored in a table in row-wise layout (NSM).

We compare the Optimistic SUM against the full 128-bit sum. Figure 11 plots the average runtime of each aggregation method against the input values. We have separated two cases: (a) the full-blown optimistic Sum and (b) if one can prove, using Min/Max information, the absence of negative values, one can optimize the SUM further. Regardless of the amount of groups in the aggregation (4 or 1024), it can be observed that (positive-only) Optimistic sum significantly outperforms the full sum for positive inputs and for values $\leq 2^{61}$. The Optimistic sum's performance heavily depends on the number of exceptions as for each exception it requires two reads, two writes, two additions, as well as two/three branches. For 4 groups the Optimistic SuM is slower than full SUM, still the optimized version for only positive inputs is the fastest for values up to $2^{61}$. For 1024 groups we observed that the Optimistic sum in both flavors, outperforms the full sum by $2 \times$. This is caused by more efficient memory access (less reads and writes).

\section{RELATED WORK}

In this paper we aim at improving cache-efficiency of hash tables through compression. An alternative approach is to increase the fill-rate using techniques such as Robin Hood Hashing [8] and Cuckoo Hashing [23]. Similarly, Concise Hash Tables [4] are optimized linear hash tables which try to omit the storage of empty rows. A major disadvantage of Concise Hash Tables is the restriction to linear hash tables whereas our techniques can be applied to linear, as well as bucket-chained hash tables. An extension of Concise Hash



(a) 4 groups

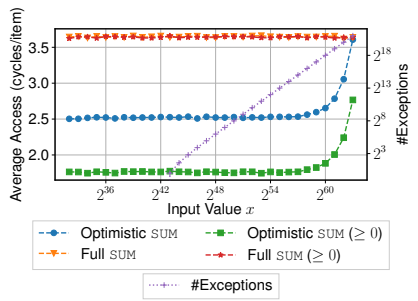

(b) 1024 groups
Fig. 11: Aggregation methods for 128-bit sum aggregate with 64-bit integers as input

Tables are Concise Array Tables [4] which avoid storing the keys by providing a collision-free hash table. These four techniques are orthogonal to our approach of treating the hash table as a compressed table. We highlight that these approaches can be applied, in addition to our techniques, to achieve even more cache-efficient hash tables.

The use of compression is widely spread among database systems. Commonly, analytic databases utilize lightweight compression schemes [33] to elevate effective memory and disk bandwidth during table scans. Although they used similar techniques as ours, notably bit-packing, frame-of-reference and dictionary-compression, in this work, we apply compression to hash tables, data structures used during query evaluation rather than in data storage. An application of compression which has not received much attention.

Graefe and Shapiro [12] discuss the applicability of data compression during query evaluation. The possibility of exploiting compressed data inside data processing pipelines, so called compressed execution, is investigated by Abadi et al. [2]. However, the application of on-the-fly compression inside hash tables, as we do, is not investigated. Łuszczak [21] investigated the integration of compressed execution into vectorized database systems and proposed the use of on-the-fly dictionaries. However, implementation and further investigation of on-thefly dictionaries were deferred to future work.

Evaluating predicates on compressed data has quite deeply been explored for table scans. Notable examples are Column Sketches [13], BitWeaving [20], ByteSlice [11], Data Blocks [15] and SIMD-Scan [32]. However, these works focus on scans in isolation.

Lee et al. [18] pioneered joins on data encoded using percolumn on-the-fly dictionaries. These dictionaries might seem closely related to the USSR. However there are two major differences: (1) The use of multiple dictionaries necessitates a translation from one encoded join column to the encoding of the other. The USSR, being a unified query-wide dictionary, does not require such a translation. (2) To fit into cache the USSR has a small fixed size, as opposed to the on-the-fly dictionaries that can grow very large.

Many database systems allow evaluating simple predicates directly on compressed data. Notable examples are IBM BLU [26], SQLServer [17], SAP HANA [10], C-Store [30], Oracle [25], Quickstep [24], and HyPer [15]. But only very few systems exploit compressed data inside query pipelines. 
Most notably IBM BLU [26] supports operations on compressed data. It performs joins on encoded and partitioned data similar to Lee et al. [18] and, hence, suffers from the same disadvantages (translating dictionary codes, large dictionaries).

Accelerating group-by/aggregation through pre-aggregation has been explored deeply [16], [19], [26]. However, preaggregation requires temporal key locality which is not a prerequisite for Optimistic Aggregates, as the latter ones rely on the domain of the values.

Shatdal et al. [29] proposed to optimize algorithms for cache efficiency. One of their techniques, key extraction in sorting/partitioning shares some similarity with Optimistic Splitting. However, Optimistic Aggregates are continuously updated, whereas extracted keys stay constant.

Encoding strings inside dictionaries has been explored by Färber et al. [10] and Binnig et al. [5]. However, both assume global dictionaries, which we do not require (Section IV-A). In contrast, the USSR is a small query-wide on-the-fly dictionary which only encodes frequent strings and avoids expensive update and delete operations.

\section{SUMmarY}

Hash tables are crucial data structures for modern query engines. However, in analytical queries they consume a significant amount of memory. We argue that shrinking hash tables not only lowers the memory footprint but also leads to faster access by fitting more data into faster memory. We argue that optimistically compressing hash tables, using Domain-Guided Prefix Suppression, Optimistic Splitting and the USSR, not only lowers the memory footprint but also leads to faster access by fitting more data into faster memory.

We implemented these techniques in the industrial-strength DBMS Vectorwise. In our experiments we found that our techniques can improve query performance by up to $25 \times$ in string-intensive queries. On the synthetic TPC-H benchmark we noticed up to $4 \times$ smaller hash tables and improved query runtime by up to $50 \%$ whereas in the (real-life) Tableau Public BI workload we achieved improvements up to $2.2 \times$.

\section{REFERENCES}

[1] D. Abadi, P. Boncz, S. Harizopoulos, S. Idreos, and S. Madden The design and implementation of modern column-oriented database systems. Foundations and Trends in Databases, 5(3):197-280, 2013.

[2] D. Abadi, S. Madden, and M. Ferreira. Integrating compression and execution in column-oriented database systems. In SIGMOD, pages 671-682, 2006

[3] C. Balkesen, J. Teubner, G. Alonso, and M. T. Özsu. Main-memory hash joins on multi-core CPUs: Tuning to the underlying hardware. In ICDE, pages 362-373, 2013.

[4] R. Barber, G. Lohman, I. Pandis, V. Raman, R. Sidle, G. Attaluri, N. Chainani, S. Lightstone, and D. Sharpe. Memory-efficient hash joins. PVLDB, 8(4):353-364, 2014

[5] C. Binnig, S. Hildenbrand, and F. Färber. Dictionary-based orderpreserving string compression for main memory column stores. In SIGMOD, pages 283-296, 2009.

[6] P. Boncz, A.-C. Anatiotis, and S. Kläbe. JCC-H: Adding join crossing correlations with skew to TPC-H. In TPCTC, pages 103-119, 2017.

[7] P. Boncz, M. Zukowski, and N. Nes. MonetDB/X100: Hyper-pipelining query execution. In $C I D R$, pages 225-237, 2005.

[8] P. Celis. Robin Hood Hashing. PhD thesis, University of Waterloo, 1986.
[9] A. Crolotte and A. Ghazal. Introducing skew into the TPC-H benchmark. In TPCTC, pages 137-145, 2012.

[10] F. Färber, S. K. Cha, J. Primsch, C. Bornhövd, S. Sigg, and W. Lehner. SAP HANA database: Data management for modern business applications. SIGMOD Record, pages 45-51, 2012.

[11] Z. Feng, E. Lo, B. Kao, and W. Xu. Byteslice: Pushing the envelop of main memory data processing with a new storage layout. In SIGMOD, pages 31-46, 2015.

[12] G. Graefe and L. D. Shapiro. Data compression and database performance. In Symposium on Applied Computing, pages 22-27, 1991.

[13] B. Hentschel, M. S. Kester, and S. Idreos. Column Sketches: A scan accelerator for rapid and robust predicate evaluation. In SIGMOD, pages $857-872,2018$.

[14] S. Jain, D. Moritz, D. Halperin, B. Howe, and E. Lazowska. SQLShare: Results from a multi-year SQL-as-a-service experiment. In SIGMOD, pages 281-293, 2016

[15] H. Lang, T. Mühlbauer, F. Funke, P. Boncz, T. Neumann, and A. Kemper. Data Blocks: Hybrid OLTP and OLAP on compressed storage using both vectorization and compilation. In SIGMOD, 2016.

[16] P. Larson. Data reduction by partial preaggregation. In ICDE, pages 706-715, 2002

[17] P. Larson, C. Clinciu, C. Fraser, E. N. Hanson, M. Mokhtar, M. Nowakiewicz, V. Papadimos, S. L. Price, S. Rangarajan, R. Rusanu, and M. Saubhasik. Enhancements to SQL server column stores. In SIGMOD, pages 1159-1168, 2013.

[18] J.-G. Lee, G. Attaluri, R. Barber, N. Chainani, O. Draese, F. Ho, S. Idreos, M.-S. Kim, S. Lightstone, G. Lohman, K. Morfonios, K. Murthy, I. Pandis, L. Qiao, V. Raman, V. K. Samy, R. Sidle, K. Stolze, and L. Zhang. Joins on encoded and partitioned data. PVLDB, 7(13):1355-1366, 2014.

[19] V. Leis, P. Boncz, A. Kemper, and T. Neumann. Morsel-driven parallelism: A NUMA-aware query evaluation framework for the manycore age. SIGMOD, pages 743-754, 2014.

[20] Y. Li and J. Patel. BitWeaving: Fast scans for main memory data processing. In SIGMOD, pages 289-300, 2013.

[21] A. Łuszczak. Simple solutions for compressed execution in vectorized database system. Master's thesis, Vrije Universiteit Amsterdam, 2011.

[22] I. Müller, C. Ratsch, and F. Färber. Adaptive string dictionary compression in in-memory column-store database systems. In EDBT, pages 283-294, 2014.

[23] R. Pagh and F. Rodler. Cuckoo hashing. J. Algorithms, 51(2):122-144, May 2004.

[24] J. Patel, H. Deshmukh, J. Zhu, N. Potti, Z. Zhang, M. Spehlmann, H. Memisoglu, and S. Saurabh. Quickstep: A data platform based on the scaling-up approach. PVLDB, 11(6):663-676, 2018.

[25] M. Poess and D. Potapov. Data compression in Oracle. In $V L D B$, pages 937-947, 2003.

[26] V. Raman, G. Attaluri, R. Barber, N. Chainani, D. Kalmuk, V. Kulandaisamy, J. Leenstra, S. Lightstone, S. Liu, G. Lohman, T. Malkemus, R. Mueller, I. Pandis, B. Schiefer, D. Sharpe, R. Sidle, A. Storm, and L. Zhang. DB2 with BLU acceleration: So much more than just a column store. PVLDB, 6(11):1080-1091, 2013.

[27] B. Răducanu, P. Boncz, and M. Zukowski. Micro adaptivity in Vectorwise. In SIGMOD, pages 1231-1242, 2013.

[28] S. Schuh, X. Chen, and J. Dittrich. An experimental comparison of thirteen relational equi-joins in main memory. In SIGMOD, pages 19611976, 2016.

[29] A. Shatdal, C. Kant, and J. F. Naughton. Cache conscious algorithms for relational query processing. VLDB, pages 510-521, 1994.

[30] M. Stonebraker, D. Abadi, A. Batkin, X. Chen, M. Cherniack, M. Ferreira, E. Lau, A. Lin, S. Madden, E. O'Neil, P. O'Neil, A. Rasin, N. Tran, and S. Zdonik. C-Store: A column-oriented DBMS. In $V L D B$, pages 553-564, 2005

[31] A. Vogelsgesang, M. Haubenschild, J. Finis, A. Kemper, V. Leis, T. Muehlbauer, T. Neumann, and M. Then. Get real: How benchmarks fail to represent the real world. In DBTEST, 2018.

[32] T. Willhalm, N. Popovici, Y. Boshmaf, H. Plattner, A. Zeier, and J. Schaffner. SIMD-scan: Ultra fast in-memory table scan using onchip vector processing units. PVLDB, 2(1):385-394, 2009.

[33] M. Zukowski, S. Heman, N. Nes, and P. Boncz. Super-scalar RAM-CPU cache compression. In ICDE, 2006.

[34] M. Zukowski, N. Nes, and P. Boncz. DSM vs. NSM: CPU performance tradeoffs in block-oriented query processing. In $D a M o N$, pages 47-54, 2008. 